\title{
Filigrane
}

Écoutes psychanalytiques

\section{Redonner la parole au sujet. Une proposition de travail à court terme, dans un contexte institutionnel}

\section{Patricia Riaño}

Volume 23, numéro 2, automne 2014

Qu'est la psychanalyse devenue ? Partie 2

URI : https://id.erudit.org/iderudit/1028921ar

DOI : https://doi.org/10.7202/1028921ar

Aller au sommaire du numéro

Éditeur(s)

Revue Santé mentale au Québec

ISSN

1192-1412 (imprimé)

1911-4656 (numérique)

Découvrir la revue

Citer cet article

Riaño, P. (2014). Redonner la parole au sujet. Une proposition de travail à court terme, dans un contexte institutionnel. Filigrane, 23(2), 27-37.

https://doi.org/10.7202/1028921ar
Résumé de l'article

L'analyste travaillant en institution peut s'inspirer du travail réalisé pendant les « entretiens préliminaires à l'analyse " pour redonner la parole au sujet en dispositifs à court terme, tout en respectant les conditions de la position de l'analyste établies par Freud et Lacan. Cette proposition a été mise en place par l'auteure notamment dans un projet au sein d'un CSSS. 


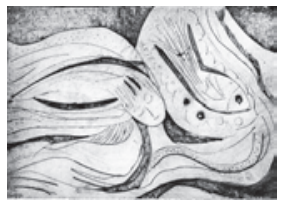

\title{
Redonner la parole au sujet. Une proposition de travail à court terme, dans un contexte institutionnel
}

Patricia Riaño

\begin{abstract}
L'analyste travaillant en institution peut s'inspirer du travail réalisé pendant les «entretiens préliminaires à l'analyse» pour redonner la parole au sujet en dispositifs à court terme, tout en respectant les conditions de la position de l'analyste établies par Freud et Lacan. Cette proposition a été mise en place par l'auteure notamment dans un projet au sein d'un CSSS ${ }^{1}$.
\end{abstract}

a proposition présentée dans cet article est le résultat de la pratique institutionnelle développée en essayant de répondre aux questions que je me suis posées comme psychanalyste intégrant le système public de santé et des services sociaux québécois, dans lequel j’ai travaillé pendant 15 ans. Certaines interrogations ont orienté mes choix. Comment peut travailler un psychanalyste dans une institution où le mandat est de faire des suivis à court terme, tout en respectant la position analytique? Comment ne pas déformer les fondements de la psychanalyse tout en sachant que la psychanalyse ne peut se pratiquer en institution? Comment s'inspirer du dispositif créé par Freud tout en reconnaissant les limites que l'institution impose?

La lecture de Freud, Winnicott et Lacan m'a aidée à trouver des réponses à ces questions. Je débuterai par Winnicott, car il a partagé son temps entre la consultation privée, où il pratiquait la psychanalyse, et le travail institutionnel. Cela lui a permis de créer une conception souple des possibilités d'intervention d'un psychanalyste. Riches en exemples concrets, ses textes montrent qu'il se permettait de faire des suivis à très court terme, adaptés aux conditions de la personne qui le consultait.

Dans Les visées du traitement psychanalytique, il dit clairement: «Si notre but continue d'être la verbalisation de la conscience qui s'éveille, en fonction du transfert, c'est bien de pratique analytique qu'il s'agit. Sinon, nous 
sommes des analystes pratiquant quelque chose que nous jugeons approprié à la situation. Et pourquoi pas?» (Winnicott, 1962, 138). Soulignons que dans cette citation, Winnicott identifie le travail du transfert comme la clé pour différencier la psychanalyse des autres possibilités d'action de l'analyste. Voilà une piste intéressante pour orienter le travail institutionnel. Dans celui-ci, il ne s'agirait donc pas du travail du transfert, même si le clinicien est un analyste. Cette alternative exige que l'on différencie clairement le travail du transfert, propre à l'analyse, d'un travail préalable, avant l'installation du transfert analytique. Ce premier temps est constitué des «entretiens préliminaires» dont on peut s'inspirer pour accomplir un travail institutionnel à court terme. Avant d'aborder celui-ci, il est toutefois nécessaire de bien distinguer deux temps: les entretiens préliminaires et l'analyse proprement dite. C'est bien l'apparition du transfert analytique qui marque le passage entre les deux, d'où l'importance de bien établir ce qui le caractérise. Le «travail du transfert» en tant que tel appartient au deuxième temps tel que présenté par Freud dans La dynamique du transfert (1912) et Observations sur l'amour de transfert (1914), ainsi que par Lacan dans le cadre du séminaire de 1960 et 1961, puis lors du séminaire de 1964, Les quatre concepts fondamentaux de la psychanalyse.

Chez Freud, il est clair que le transfert analytique n'existe pas dans le premier temps du travail; il faut créer les conditions pour qu'il apparaisse. Et quand cela arrive, il s'opère une transition de la «névrose ordinaire» vers la «névrose de transfert». Dans le texte Le début du traitement, Freud écrit que le moment opportun pour révéler à l'analysant la signification secrète de ses pensées suit l'établissement d'un transfert «sûr ${ }^{2}$ », nom qu'il donne à ce type de transfert dans ce texte. Il propose certaines indications pour que le patient parvienne à inclure le médecin dans l'une des imagos des personnes qui dans le passé lui avaient donné de l'amour (1913). Dans Remémoration, répétition, perlaboration, il parle de ce passage entre la névrose ordinaire et la névrose de transfert en termes d'un changement dans les symptômes, de la possibilité de donner aux symptômes une signification transférentielle (1914) qui opère un changement fondamental dans ceux-ci. Le symptôme, par sa nature, n'est dirigé vers personne. On se rappellera de la définition classique donnée par Freud du symptôme comme formation de compromis entre une manifestation pulsionnelle inacceptable et la défense contre celle-ci (1896). Le symptôme est l'exercice d'un plaisir à la fois méconnu et interdit. Dans l'analyse, à travers le transfert, le symptôme sera dorénavant adressé à l'analyste, celui-ci y sera inclus. 
Lacan soutient la même position que Freud quant au transfert analytique. Il parle d'une séquence ordonnée qui commence par la «rectification subjective» qui permet ensuite l'installation du transfert; l'interprétation ne peut précéder l'établissement du transfert, elle doit lui succéder. En effet, Lacan considérait que la proposition d'interprétations avant que le transfert ne soit établi était une erreur. Dans La direction de la cure et les principes de son pouvoir (1966), en discutant la manière de procéder de Freud, Lacan souligne: « $[\ldots]$ une direction de la cure s'ordonne, $[. .$.$] selon un procès qui$ va de la rectification des rapports du sujet avec le réel, au développement du transfert, puis à l'interprétation [...]» (p. 598).

Le premier moment souligné par Lacan est donc celui de la «rectification des rapports du sujet avec le réel» ou comme lui-même l'appelait aussi «rectification subjective». Insistons sur ce point. Il ne s'agit pas encore de transfert analytique et encore moins d'interprétation. De quoi s'agit-il donc dans la rectification subjective? On invite le sujet à reconnaître sa participation, souvent inconsciente, dans la création ou le maintien des situations qui le font souffrir, soit ce que le sujet met en jeu et les raisons de ce faire. En d'autres termes, on invite le sujet à prendre la parole, à parler de ce qui lui arrive, à parler de sa compréhension. Dans La direction de la cure, Lacan propose d'aider le sujet à faire un «repérage de sa position dans le réel», à la manière de Freud au début de ses traitements. Il y illustre bien la rectification subjective que Freud a accomplie chez Dora, en travaillant la participation active que celle-ci avait dans le complexe de relations en quartette entre elle, son père, $M$. et $\mathrm{M}^{\mathrm{me}} \mathrm{K}$. : «Autre exemple notoire: quand il [Freud] réduit Dora à constater que ce grand désordre du monde de son père, dont le dommage fait l'objet de sa réclamation, elle a fait plus que d'y participer, qu'elle s'en était faite la cheville et qu'il n'eût pu se poursuivre sans sa complaisance» $(1966,596)$.

Le second moment identifié par Lacan est celui du développement du transfert. Très tôt dans son enseignement, Lacan conseillait aux analystes de ne pas s'accrocher aux passions de l'amour et de la haine. Il proposait d'écouter aussi, et surtout, la passion de l'ignorance: «Un tel schéma [Lacan fait ici référence à un schéma de deux pyramides triangulaires unies et opposées par leurs bases, qu'il a montré dans cette séance du séminaire] vous présentifie ceci - c'est seulement dans la dimension de l'être, et non pas dans celle du réel, que peuvent s'inscrire les trois passions fondamentales — à la jonction du symbolique et de l'imaginaire, cette cassure, si vous voulez, cette ligne d'arête qui s'appelle l'amour — à la jonction de l'imaginaire et du réel, 
la haine — à la jonction du réel et du symbolique, l'ignorance. Nous savons que la dimension du transfert existe d'emblée, implicitement, avant tout commencement de l'analyse, avant que le concubinage qu'est l'analyse ne le déclenche. Or, ces deux possibilités de l'amour et de la haine ne vont pas sans cette troisième, qu'on néglige, et qu'on ne nomme pas parmi les composantes primaires du transfert - l'ignorance en tant que passion. Le sujet qui vient en analyse se met pourtant, comme tel, dans la position de celui qui ignore. Pas d'entrée possible dans l'analyse sans cette référence - on ne le dit jamais, on n'y pense jamais, alors qu'elle est fondamentale» (19531954, 297-298).

Le travail de rectification subjective réalisé pendant les entretiens préliminaires est propice à un changement du sujet par rapport à cette passion de l'ignorance. De la position de ne «rien vouloir savoir» du départ, où la jouissance ${ }^{3}$ domine le tableau, se dégage un désir de savoir sur sa propre souffrance. Alors, une ou quelques questions apparaissent, par exemple: Pourquoi cela m'arrive-t-il? Pourquoi je me retrouve toujours dans les mêmes situations?, etc. Ces questions sont adressées à l'analyste dont le sujet suppose qu'il détient le savoir qui lui manque. L'analysant a ainsi institué le Sujet supposé Savoir (S.s.S.). Lacan (1964) affirme que dès qu'il y a sujet supposé savoir, il y a transfert: «La question est d'abord, pour chaque sujet, d'où il se repère pour s'adresser au sujet supposé savoir. Chaque fois que cette fonction peut être, pour le sujet, incarnée dans qui que ce soit, analyste ou pas, il résulte de la définition que je viens de vous donner que le transfert est d'ores et déjà fondé» (p. 211). Ce qui marque la différence entre l'analyse et d'autres situations, est fondamentalement la position de l'analyste et la façon dont il travaille sachant que ce savoir lui est attribué. D’un autre côté, Lacan montre bien que «la mise en action du transfert» dans l'analyse prend du temps à se déployer: "L'analyste, vous ai-je dit, tient cette place [S.s.S.] pour autant qu'il est l'objet du transfert. L'expérience nous prouve que le sujet, quand il entre dans l'analyse est loin de lui donner cette place» (p. 211).

Suite à cette brève révision, revenons à l'interrogation initiale sur la possibilité, pour un analyste, de travailler en institution. Tout dans ce qui précède semble démontrer que le travail du transfert ne peut s'accomplir au sein d'une institution. D'un côté, il y a la dimension temporelle: la limitation de la durée des suivis thérapeutiques, ce qu'on appelle les interventions à court terme, empêche le déploiement du transfert. Mais, même dans le cas hypothétique où une institution enlèverait cette restriction, le travail voire même l'analyse du transfert serait encore entravée par d'autres contraintes 
inhérentes au travail en institution. En effet, tout «intervenant» (terme par lequel sont désignés les professionnels dans ce contexte) travaillant dans une institution la représente; le transfert serait forcément traversé, coloré par cette réalité. L'analyste en institution n'a pas la liberté d'action nécessaire à l'analyse. Il doit se soumettre à des directives, à de constants changements de politique, aux exigences administratives qui proviennent du grand Autre institutionnel, qu'il ne peut souvent ni contrôler ni contester.

Confronté à cet impossible travail du transfert dans l'institution, quel rôle peut donc y jouer un analyste? Comme je l'énonçais plus haut, l'avenue pour le travail institutionnel à court terme serait, à mon avis, de s'inspirer des entretiens préliminaires, ou plus précisément, de la «rectification subjective» ou «rectification des rapports avec le réel» proposée par Lacan. Celui-ci proposait un «repérage de la position du sujet»; ainsi, l'analyste en institution aiderait le sujet à reconnaître les jeux relationnels auxquels il participe et à identifier la jouissance qui accompagne ses symptômes.

De quelle manière peut-on procéder dans ce travail de rectification subjective si, tel que mentionné précédemment, l'interprétation en est exclue? Plusieurs interventions permettent de respecter les conditions de la position de l'analyste et de garantir que la parole soit redonnée au sujet. Voyons quelques éléments concrets qui sous-tendent la position de l'analyste.

- Donner la parole au sujet. On essaie donc de donner la place au sujet, de l'écouter, et on évite de «renforcer le moi $^{4} »$. Dans Fonction et champ de la parole et du langage en psychanalyse (1966), Lacan fait une distinction entre "parole pleine» et "parole vide». Sur celle-ci, il déclare: «Nous avons abordé la fonction de la parole dans l'analyse par son biais le plus ingrat, celui de la parole vide, où le sujet semble parler en vain de quelqu'un qui, lui ressemblerait-il à s'y méprendre, jamais ne se joindra à l'assomption de son désir» (p. 254). C'est une parole où le sujet n'a pas une place de protagoniste; il serait là oui, dans ce discours, mais effacé. Tandis que dans la "parole pleine», il s'agit de la vérité du sujet. Dans ce même texte, Lacan affirme: «Soyons catégorique, il ne s'agit pas dans l'anamnèse psychanalytique de réalité, mais de vérité, parce que c'est l'effet d'une parole pleine de réordonner les contingences passées en leur donnant le sens des nécessités à venir, telles que les constitue le peu de liberté par où le sujet les fait présentes» (p. 256). Cette distinction est bien résumée dans le séminaire Les écrits techniques de Freud (1953-1954): «En fin de compte, ce à quoi nous sommes ramenés par cette considération, 
n'est-ce pas ce dont je suis parti dans mon rapport sur les fonctions de la parole? à savoir l'opposition de la parole vide et de la parole pleine, parole pleine en tant qu'elle réalise la vérité du sujet, parole vide par rapport à ce qu'il a à faire hic et nunc avec son analyste, où le sujet s'égare dans les machinations du système du langage, dans le labyrinthe des systèmes de référence que lui donne l'état culturel où il a plus ou moins partie prenante. Entre ces deux extrêmes, se déploie toute une gamme de réalisation de la parole» (p.61). Quoique difficile dans un contexte de travail à court terme, la parole pleine du sujet peut avoir une chance de s'énoncer si on lui laisse la place, si on est à son écoute et si on permet que la parole vide puisse glisser vers la parole pleine à travers cette gamme de réalisations que mentionnait Lacan dans la précédente citation.

- Écouter à la lettre. Tel que Freud le conseillait dans l'interprétation des rêves, il s'agit de prendre les paroles du sujet comme un texte, une écriture. On ne parle pas ici de «l'écoute active» utilisée dans d'autres approches. Dans «écouter à la lettre» il s'agit d'une discipline qui vise à limiter l'imaginaire de l'analyste, à éviter de donner des significations au discours du sujet de façon hâtive. C'est une invitation, tel que le suggérait Lacan, à ne pas comprendre ou au moins, à ne pas comprendre trop vite. Sur la compréhension, il dit dans le séminaire Les écrits techniques de Freud (1953-1954): «Combien de fois ne l'aije pas fait observer à ceux que je contrôle quand ils me disent - J'ai cru comprendre qu'il voulait dire ceci, et cela - une des choses dont nous devons le plus nous garder, c'est de comprendre trop, de comprendre plus que ce qu'il y a dans le discours du sujet. Interpréter et s'imaginer comprendre, ce n'est pas du tout la même chose. C'est même exactement le contraire. Je dirais même que c'est sur la base d'un certain refus de compréhension que nous poussons la porte de la compréhension analytique» (p. 87). Plus tard, dans le séminaire Le transfert (1960-1961) il le confirme: "Il faut qu'il [l'analyste] sache en particulier que le critère de sa position correcte n'est pas qu'il comprenne ou qu'il ne comprenne pas. Il n'est pas absolument essentiel qu'il comprenne. Je dirai même que, jusqu'à un certain point, qu'il ne comprenne pas peut être préférable à une trop grande confiance dans sa compréhension. En d'autres termes, il doit toujours mettre en doute ce qu'il comprend, et se dire que ce qu'il cherche à atteindre, c'est justement ce qu'en principe, il ne comprend pas» (p. 234). 
- L'analyste ne reste pas silencieux. Si les dires du sujet sont un texte à lire, on peut l'aider dans son travail de ponctuation lorsqu'il écoute son propre récit (Lacan, 1966, 252). On peut questionner, montrer les contradictions et, même si l'interprétation est exclue dans ce temps de la rectification subjective, on peut, par exemple, souligner les idées qui reviennent, interroger celles qui le font réagir, ou celles où une émotion se présente.

- Le savoir est du côté du sujet. Face aux connaissances proposées par tous les moyens (professionnels, Internet, médias), le sujet est invité à écouter son savoir sur lui-même. Cela implique que l'analyste ne se propose pas comme expert, le savoir n'est donc pas de son côté, et ne saurait être exproprié au sujet.

- L'approche éducative est exclue. La position de l'analyste n'est pas celle d'un enseignant; il n'a rien à apprendre au sujet. Il lui offre, par contre, les conditions pour interroger son désir. Ce faisant, il ne peut endosser le rôle de modèle pour le sujet.

- En conséquence, il est important de souligner que l'analyste ne peut déterminer ce qui est «le bien» du sujet et le lui imposer, à partir de ses propres valeurs. Lacan a d'ailleurs dédié une partie importante dans son séminaire L'éthique de la psychanalyse (1959-1960) à cette question du «bien». Le psychanalyste n'est donc pas en position de surmoi, ce qui constitue une application évidente de ce que Freud a conceptualisé comme la règle de l'abstinence pour l'analyste (1915 [1914], 168).

- Dans le cadre de l'institution, le psychanalyste travaille à partir de la demande initiale. Il tente de différencier celle du sujet de celle du médecin ou de tout autre référant, si tel est le cas, et de se centrer sur la demande du sujet. En travaillant la demande, les plaintes, il ouvre la voie à ce que le sujet puisse parler de ses interrogations, de ce qui le dérange. C'est une expérience commune à tous les cliniciens que la demande initiale puisse faire place à des préoccupations qui n'ont souvent jamais été manifestées auparavant, à des secrets jamais partagés. Apparaissent ainsi les thèmes qui étaient cachés derrière la demande première, ou articulés à elle, et sur lesquels portera le travail des séances.

- Inspiré par la règle fondamentale, le psychanalyste invite le sujet à parler sans trop juger ce qu'il dit et, s'il le fait, à exprimer tant le jugement que le contenu jugé. Il l'invite aussi à prêter attention aux pensées spontanées apparues entre les séances, en continuité avec le travail inhérent 
aux rencontres. Il l'invite à éviter la « réflexion» comme activité volontaire de «se mettre» à penser. Illustrons ce point avec un exemple.

Entre la première et la deuxième séance, une jeune femme qui consulte pour avoir des outils contre les crises d'angoisse «écoute» pour la première fois les pensées qui la réveillent. Ces pensées auxquelles elle ne prêtait pas attention malgré qu'elles fussent toujours présentes en «arrière-plan », prenaient toute la place pendant ses nuits d'insomnie. Elle découvre qu'elle a peur de la solitude et cela la surprend, car elle a un conjoint ainsi qu'une fille, et la relation qu'elle entretient avec eux est très bonne, de même qu'avec toute sa famille et ses amis. La « découverte» de la peur de la solitude, faite lors d'une nuit d'insomnie, a permis qu'à partir de la deuxième séance elle se mette à parler de sa peur de la mort, puis d'une phobie de la noirceur développée pendant l'enfance, mais encore présente.

- Le psychanalyste permet que le sujet fasse des liens avec son histoire. Même en l'absence d'une demande explicite, la plupart des sujets repèrent spontanément des connexions entre la situation présente qui les dérange et leur histoire personnelle et familiale. Souvent des éléments de répétition sont identifiés.

De façon générale, ce type d'intervention facilite le changement de position du sujet, de la plainte initiale au travail sur sa souffrance. Ainsi est véritablement redonnée la parole au sujet, parole qui a été tue pour toutes sortes de raisons, dont la jouissance que procure le symptôme. En conséquence, le sujet peut dès lors faire le passage de «la parole vide» qui se perd dans le bavardage et qui reste dans des circuits sans issue, vers «la parole pleine», celle qui conduit à l'action, qui ouvre la possibilité du changement, du passage d'une position subjective de «subir» à une position plus active quant aux décisions qui le concernent. Pour illustrer la possibilité d'un changement de position du sujet, j'ai choisi l'exemple d'une femme qui a été référée par son médecin suite à un diagnostic de dépression. C'était aussi son motif de consultation.

Dans la deuxième séance, elle se plaint beaucoup de sa fille et de son gendre. Ils abusent d'elle, de son temps et de son argent. Sans le vouloir, elle a pris la responsabilité des paiements de leurs meubles et de leur camion. Elle paie le linge des enfants et l'épicerie, mais elle n'a pas d'argent 
pour ses propres dépenses. Elle doit «compter ses sous» pour s'acheter du pain et du lait, seule nourriture qu'elle s'est offerte pendant les mois les plus difficiles, pendant que sa fille louait un chalet dans le Nord, pour les vacances. Dans ce cas-ci, le travail de rectification subjective, de repérage de sa position, a consisté à rediriger ses plaintes continuelles envers son gendre, vers ses propres motivations qui la faisaient tomber à répétition dans ces pièges. Madame a reconnu qu'en faisant cela, elle achetait l'amour. Jamais elle ne s'est sentie digne de l'amour ou de l'attention des autres. Elle comble toutes les demandes qu'on lui adresse et souvent les anticipe en donnant avant même qu'on la sollicite. Pour se faire aimer, elle est prête aux plus grands sacrifices. À partir de la reconnaissance de sa participation dans la situation qu'elle déplore, un changement de position de sa part est amorcé: le travail est dirigé vers cette problématique de l'amour, plutôt que d'attendre un impossible changement de comportement de son gendre, comportement qu'en fait elle encourageait et maintenait.

Prendre comme modèle les entretiens préliminaires, avec le travail de rectification de la position subjective qu'ils impliquent, m'a permis de construire une approche à court terme qui réponde positivement à la possibilité de maintenir une position analytique dans le cadre et les limites que l'institution détermine. En effet, cette position peut être maintenue même s'il ne s'agit pas d'arriver, dans ce contexte, au travail du transfert tel que défini ci-dessus en suivant les pistes ouvertes par Freud, Winnicott, puis Lacan. En fait, je considère que cela représente une alternative à une certaine tendance d'uniformisation de l'intervention dans le système de santé et des services sociaux où la psychoéducation prend une place de plus en plus importante, au détriment des modèles thérapeutiques qui reconnaissent et respectent la singularité du sujet.

C'est donc avec cette approche que j'ai travaillé pendant 15 ans dans des institutions du système de santé et des services sociaux du Québec. Et c'est aussi l'approche que j'ai déployée dans le cadre d'un projet, à l'élaboration duquel j’ai participé, au sein du CSSS de Laval ${ }^{5}$ pendant cinq ans. En voici une brève description.

Sachant que, selon la RAMQ ${ }^{6}$, autour de 30 ou $40 \%$ des consultations des médecins en cliniques médicales portent sur des problèmes de santé mentale, le ministère a demandé au CSSS de créer un programme pour appuyer les médecins. Nous avons établi que nous recevrions les références, de façon presque immédiate, des personnes ayant un diagnostic médical de 
troubles anxieux, dépressifs, ou d'adaptation avec une composante anxieuse ou dépressive. Un maximum de six séances est offert. Si ce nombre s'avère insuffisant, «le client» (nom donné par le système aux usagers) est référé à la liste d'attente du $\mathrm{CLSC}^{7}$ pour une psychothérapie, ou à tout autre service jugé approprié. Pendant ces cinq ans, j’y ai travaillé à temps partiel, à raison d'une ou deux journées par semaine. J'ai reçu plus de 200 clients dont la grande majorité a mis fin au suivi après six séances ou moins; seulement $7 \%$ des sujets ont été référés après les six séances offertes. Les «clients» ont trouvé ce travail très aidant. Au-delà de l'évaluation immédiate effectuée lors de la dernière séance, une évaluation plus formelle a été réalisée à l'aide d'un questionnaire construit à cette fin et validé par plusieurs instances du CSSS dont le Comité des usagers. Le questionnaire pouvait être complété anonymement. Il était envoyé par la poste à l'une des agentes administratives de la Direction de Santé mentale. Un des résultats collatéraux de cette formule est le changement d'habitudes repéré chez certains médecins. Constatant l'effet de ce travail sur leurs patients, dans certains cas, ils ont préféré retarder, limiter ou exclure la prescription de médicaments, en attendant l'évolution de l'état de leur patient suite aux séances du programme.

\section{Conclusion}

Une façon de travailler à court terme inspirée par la psychanalyse, et plus particulièrement par le travail développé au cours des entretiens préliminaires, serait donc possible. Dans le contexte institutionnel, il est envisageable de réaliser un travail rigoureux et sérieux sans déroger de la position de l'analyste. Cette position se met en place à partir des premières séances, même si le travail ne conduit pas à une analyse en tant que telle, même s'il n’y a pas transfert analytique à proprement parler, moment qui marquerait l'entrée en analyse. Le maintien de cette position garantit la mise en place des conditions nécessaires pour l'écoute de la parole du sujet, dans sa singularité. C'est une approche qui représente une alternative efficace à une tendance, de plus en plus présente, à universaliser, à massifier, à uniformiser ceux qui nous consultent.

Patricia Riaño 306, rue Golfridge

Rosemère J7A 2M5 patriciariano@yahoo.com 


\section{Notes}

1. Centre de santé et de services sociaux. Au Québec, les services de santé et psychosociaux sont regroupés par régions, au sein de tels centres.

2. Dans la version consultée, Freud parle de «transferencia operativa».

3. Jouissance est un terme utilisé par Lacan pour parler de cette double allégeance du symptôme qui lui permet à la fois la satisfaction d'un plaisir méconnu et son interdiction.

4. En accord avec la théorie de Lacan, le moi — lieu des identifications imaginaires du moi idéal et plus généralement de l'imaginaire — se distingue du Sujet — qui prend racine dans l'inconscient et qui se construit en référence au symbolique. Rappelons que pour Lacan, c'est le «sujet» qui parle, c'est-à-dire le sujet de l'inconscient. Dans le séminaire Le moi dans la technique de la psychanalyse (1954-1955), il explique clairement: "Dans l'inconscient, exclu du système du moi, le sujet parle» (p. 77).

5. Ville en banlieue de Montréal.

6. Régie de l'assurance maladie du Québec; au Québec, la régie administre les régimes publics d'assurance maladie et médicaments.

7. Centre local de services communautaires, soit une partie intégrante du réseau des CSSS, qui offre des services de santé et des services sociaux à la population locale.

\section{Références}

Freud, S., 1896, Nuevas puntualizaciones sobre las neuropsicosis de defensa, in Obras completas, Tomo III, Buenos Aires, Amorrortu editores, 1981.

Freud, S., 1912, Sobre la dinámica de la transferencia, in Obras completas, Tomo XII, Buenos Aires, Amorrortu editores, 1980, 95-105.

Freud, S., 1913, Sobre la iniciación del tratamiento (Nuevos consejos sobre la técnica del psicoanálisis, I), in Obras completas, Tomo XII, Buenos Aires, Amorrortu editores, 1980.

Freud, S., 1914, Recordar, repetir y reelaborar, (Nuevos consejos sobre la técnica del psicoanálisis, II), in Obras completas, Tomo XII, Buenos Aires, Amorrortu editores, 1980.

Freud, S., 1915 [1914], Puntualizaciones sobre el amor de transferencia (Nuevos consejos sobre la técnica del psicoanálisis, III), in Obras completas, Tomo XII, Buenos Aires, Amorrortu editores, 1980, 163-174.

Freud, S., 1923, El yo y el ello, in Obras completas, tomo XIX, Buenos Aires, Amorrortu editores, 1979, 30-31.

Lacan, J., 1953-1954, Le séminaire, Livre I, Les écrits techniques de Freud, Paris, Seuil, 1975.

Lacan, J., 1954-1955, Le séminaire, livre II, Le moi dans la théorie de Freud et dans la technique de la psychanalyse, Seuil, 1978.

Lacan, J., 1959-1960, Le séminaire, livre VII, L'éthique de la psychanalyse, Paris, Seuil.

Lacan, J., 1960-1961, Le séminaire, Livre VIII, Le transfert, Paris, Seuil, 2001.

Lacan, J., 1962-1963, L'angoisse, Séminaire 1962-1963, Publication hors commerce. Document interne destiné à l'Association freudienne internationale et destiné à ses membres, Leçon du 16 mars 1963, 211.

Lacan, J., 1964, Le séminaire, livre XI, Les quatre concepts fondamentaux de la psychanalyse, Paris, Seuil, 1973.

Lacan, J., 1966, Séminaire sur «la Lettre volée», in Écrits, Paris, Seuil.

Lacan, J., 1966, La direction de la cure et les principes de son pouvoir, in Écrits, Paris, Seuil.

Lacan, J., 1966, Fonction et champ de la parole et du langage en psychanalyse, in Écrits, Paris, Seuil.

Winnicott, D. W., 1962, Les visées du traitement psychanalytique, in Processus de maturation chez l'enfant, Paris, Petite bibliothèque Payot. 